\title{
Artificial Intelligence - Where it is heading and what we should do about it
}

\author{
Artificial Intelligence (Al) is already shaping our everyday lives. While there is enormous poten- \\ tial for harnessing Al to solve complex industrial and social problems and to create new and \\ innovative products and solutions, many organisations are still grappling to understand the \\ relevance and future impact of Al on their activities and what they should be doing about it.
}

$\mathrm{AI}$ is a rapidly changing field. Recent advances in machine learning (ML), specifically deep learning, have provided practical solutions to automate complex tasks in computer vision, natural language processing, and task learning. New and improved ML techniques are being produced at an astounding rate by ML researchers. Even more incredible is that the lag time for testing and implementation of these techniques in real world applications has also decreased significantly. Many ML researchers are now publishing their results instantly on open publication platforms, such as arxiv.org, and the research community is increasingly demanding code implementations which eases reproducibility, verification and further testing. Established and freely available ML platforms such as PyTorch and TensorFlow are now widely used by both academia and industry. The gap between the ML researcher and the ML practitioner is continuously closing.

However, machine learning applications do have their limitations. Machine learning models generally do not offer explanations for their outputs, require large labelled training data sets with uniformly distributed samples, can incorporate historical biases, and do not adapt well to novel scenarios. Furthermore, while machine learning models perform well for specific tasks, ML alone is insufficient for developing autonomous systems that can adapt to changing environments. Another branch of AI, referred to as the model-based approach in a recent insightful article by Adnan Darwiche [1], has techniques that can potentially deal with many of the limitations of machine learning. Many AI experts believe that the model-based and ML approaches are complementary, and that future AI systems will incorporate techniques from both these areas.

Because of the enormous potential of AI, it is important for AI researchers, practitioners, and policy makers to get it right from the start. Without coordinated interventions, AI innovation and development will be driven from the ground up. Niche technology companies and startups will develop advanced expertise and compete to dominate the AI space. This will drive up the cost of AI and constrain AI to specialized applications that require large capital investments. Innovative AI applications will develop in an ad hoc manner and will be constrained to specific application areas.

$\mathrm{AI}$ is a rapidly changing field and most companies will not be able to establish effective in house AI capabilities to keep abreast of the pace of developments in the AI R\&D community. While large companies should at least establish some in house capacity, either an AI office or an AI champion, partnering with an AI R\&D academic or commercial partner will likely be a more effective and sustainable route for most organisations.
A broader and more long term vision is to establish local, regional or country-wide AI innovation networks. From a country perspective this will involve a long term skills development plan, accessibility paths for reskilling, and the development and maintenance of a set of tools, methods and platforms for rapid prototyping of AI techniques. Such an AI innovation ecosystem will provide a collaborative platform for academics, industry and government to guide and direct the development of AI, and provide rapid dissemination and access to advanced AI knowledge beyond Computer Science and Engineering. The establishment of such ecosystems will be crucial to accelerate innovation using $\mathrm{AI}$, reskilling and skills development, lower the cost of AI, and to make AI accessible to a broader community.

A/Prof. Deshendran Moodley and Prof. Thomas Meyer

References: [1] Darwiche, A., 2018. Human-level intelligence or animal-like abilities? Communications of the ACM, 61(10), pp.56-67.

\section{Prof. Deshen Moodley}

Deshen Moodley is an Associate Professor in the Department of Computer Science at the University of Cape Town (UCT) and co-founder and Deputy Director of the South African National Centre for Artificial Intelligence Research. Having last worked as Director of an Internet startup company, SystemsFusion, based in Denver Colorado, he returned to academia in 2001. His broad research is in Artificial Intelligence, specifically the development of systems that can self-learn and adapt to a dynamic and evolving environment.

\section{Prof. Thomas Meyer}

Prof. Thomas Meyer (born in 1964) has been the UCT-CSIR Chair in Artificial Intelligence, and a full professor in Computer Science at the University of Cape Town since 2015. In 2011 he co-founded the South African Centre for Artificial Intelligence Research (CAIR), and has been its Director since then. Thomas is recognised internationally as an expert in Knowledge Representation and Reasoning.
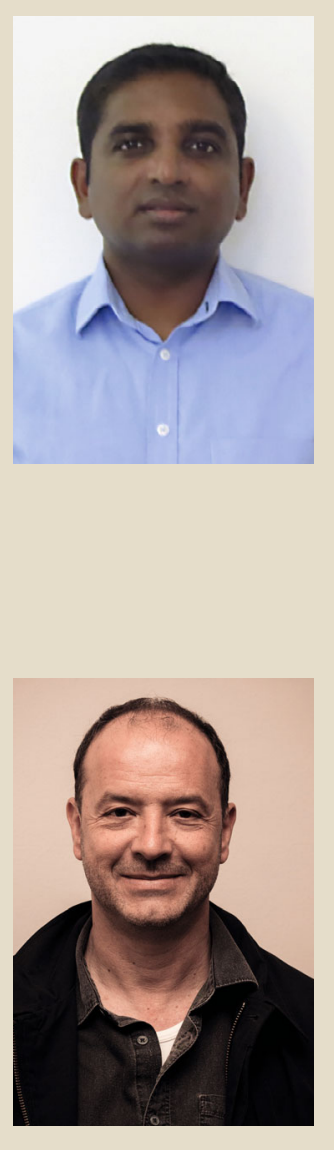